\title{
The role of microRNAs in cell death pathways
}

\author{
Ji Hoon Jang, Tae-Jin Lee \\ Department of Anatomy, Yeungnam University College of Medicine, Daegu, Korea
}

Received: November 16, 2020

Revised: December 9, 2020

Accepted: December 12, 2020

Corresponding author:

Tae-Jin Lee, PhD

Department of Anatomy, Yeungnam

University College of Medicine, 170

Hyeonchung-ro, Nam-gu, Daegu

42415, Korea

Tel: +82-53-640-6914

Fax; +82-53-621-5083

E-mail: tjlee@med.yu.ac.kr
MicroRNAs (miRNAs) are a class of noncoding RNAs that negatively regulate target messenger RNAs. In multicellular eukaryotes, numerous miRNAs perform basic cellular functions, including cell proliferation, differentiation, and death. Abnormal expression of miRNAs weakens or modifies various apoptosis pathways, leading to the development of human cancer. Cell death occurs in an active manner that maintains tissue homeostasis and eliminates potentially harmful cells through regulated cell death processes, including apoptosis, autophagic cell death, and necroptosis. In this review, we discuss the involvement of miRNAs in regulating cell death pathways in cancers and the potential therapeutic functions of miRNAs in cancer treatment.

Keywords: Apoptosis; Autophagy; Endoplasmic reticulum stress; MicroRNAs; Necroptosis

\section{Introduction}

MicroRNAs (miRNAs or miRs) are small (20-23 nucleotides) noncoding RNAs that negatively regulate messenger RNAs (mRNA) expression by blocking translation or by directly promoting degradation of the target mRNA [1]. It is estimated that miRNAs regulate approximately $30 \%$ of the protein-coding genes in humans. Individual miRNAs can regulate the expression of multiple genes, and conversely, a single target gene can be regulated by many miRNAs [1].

More than half of the miRNA genes are commonly located in introns. miRNAs are produced by endogenously transcribed long primary miRNA (pri-miRNA) by RNA polymerase (Pol) II or Pol III and show a typical mRNA form with a 5'-cap structure and a 3'poly (A) tail $[2,3]$. The pri-miRNAs are further processed into a hairpin-shaped structure, 60 to 100 nucleotides in length, known as precursor miRNA (pre-miRNA) by the nuclear RNase III Drosha. This nuclear processing event is site- specific and critical for determining the mature miRNA sequence. After being exported to the cytoplasm by double-stranded RNA-binding proteins (exportin-5), pre-miRNAs undergo a final processing event by RNase III family enzymes (Dicer) to form a 20-nucleotides miRNA duplex.
These mature miRNA duplexes are unwound and the mature strand is incorporated into the RNA-induced silencing complex (RISC) together with an Argonaute (AGO) protein, subsequently forming an active RISC complex [4]. This complex binds to specific mRNA with a complementary sequence, usually in the 3 '-untranslated regions (UTRs) of mRNAs to trigger mRNA silencing by translational repression or degradation $[5,6]$. In general, miRNAs partially base pair with the sequence of the 3'-UTRs of the target mRNAs. This partial complementarity often facilitates translational repression [7]. In contrast, perfect or near-perfect complementary miRNA-mRNA interaction induces the cleavage of mRNA through AGO endonuclease activity, exerting a strong repressive effect on target mRNA expression [8].

The modulation of miRNAs including suppressing the oncogenic miRNAs (oncomiRs) and replacing the deficient tumor-suppressive miRNAs (oncosuppressor miRs) in cancer cells may be a reliable tool to improve cancer therapy [9]. miRNAs are involved in many biological processes, such as cell proliferation, cell death, and tumorigenesis. In addition, regulation of gene expression by miRNAs is important for cellular responses to environmental stresses such as starvation, hypoxia, oxidative stress, and DNA damage. miRNAs can strongly affect the expression of various cell

Copyright (C) 2021 Yeungnam University College of Medicine

This is an Open Access article distributed under the terms of the Creative Commons Attribution Non-Commercial License (http://creativecommons.org/licenses/by-nc/4.0/) which permits unrestricted non-commercial use, distribution, and reproduction in any medium, provided the original work is properly cited. 
death-related genes such as pro- and antiapoptotic genes, autophagy regulation genes, endoplasmic reticulum (ER) stress genes, and/or necroptosis-related genes [10]. Abnormal expression of miRNAs associated with cell death pathways can affect physiological conditions and promote disease, including carcinogenesis [11].

Numerous miRNAs can function as oncogenes or tumor suppressors, and it is widely accepted that dysregulation of miRNA expression is closely related to the initiation, progression, and metastasis of cancer. An aberrant miRNA expression is a common event in malignant tissues compared to that in their normal counterparts because many miRNAs are frequently located at vulnerable sites in the human genome, causing gene amplification and deletion, chromosomal rearrangement, and epigenetic changes [11].

miRNAs are classified as oncomiRs or oncosuppressor miRs, which specifically target oncogenes and tumor-suppressor genes, respectively. Generally, oncomiRs are overexpressed, while oncosuppressor miRs are underexpressed in cancers [12]. OncomiRs are associated with specific cancer forming (oncogenic) events, including carcinogenesis, malignant transformation, and metastasis. Abnormal expression of these miRNAs favors uncontrolled proliferation and survival, promotes invasive behavior, and contributes to tumor initiation and progression by regulating cell death. Oncosuppressor miRs generally prevent tumor development by suppressing oncogenes and/or genes that control cell differentiation or cell death. Oncosuppressor miRs can be downregulated as a result of deletions, epigenetic silencing, or loss of transcription factor expression.

Despite global dysregulation, most miRNA expression is globally suppressed in cancer tissues compared to that in normal tissue counterparts [13]. Genetic deletion of the miRNA-processing machinery causes global depletion of miRNAs, which favors cell transformation and tumorigenesis in vivo [14]. This indicates that miRNA alterations play a causative role in the development of cancer, not just the effect of tumorigenesis [15]. In this review, we discuss the recent progress in the understanding of miRNA function in the regulation of apoptosis, autophagy, ER-stress-mediated cell death, and necroptosis in cancer cells.

\section{The role of microRNAs in apoptotic pathways}

Apoptosis is a programmed cell death that is precisely coordinated by genes and plays an important role in maintaining cellular homeostasis. Apoptosis occurs physiologically in cells that are no longer needed, as observed during many developmental processes. Apoptosis also occurs when cells commit a regulated suicide for the overall benefit of the organism in pathological situations such as infections, neoplasm formation, and irreversible cell damage.
The two main types of apoptotic pathways are "intrinsic pathways" and "exogenous pathways" [16,17]. The intrinsic apoptotic pathway receives signals to destroy cells from one of its genes or proteins due to the detection of DNA damage. In the extrinsic apoptotic pathway, cells receive signals from other cells in the organism or from outside the cell, instructing it to commit programmed cell death. The extrinsic and intrinsic pathways can be linked by different signaling pathways to common pathways, for example, by Bid, a $\mathrm{BH} 3$ domain-containing protein of $\mathrm{B}$-cell lymphoma 2 (Bcl-2). The truncated Bid trigger switches from the external to the internal signaling pathway [18].

The extrinsic pathway transmits extracellular death signals by the cell-surface death receptor (DR), leading to the formation of the death-inducing signaling complex (DISC) [19]. DRs belong to the tumor necrosis factor (TNF)/nerve growth factor superfamily. DRs possess the death domain (DD), death effector domain, the transmembrane domain, and cysteine-rich motifs [20]. There are six mammalian DRs (TNF receptor 1 [TNFR1], Fas, DR3, DR4 [TNF-related apoptosis-inducing ligand receptor 1, TRAILR1], DR5 [TRAILR2], and DR6) [21,22].

The intrinsic pathways are regulated by the release of cytochrome $c$ from mitochondria or loss of the mitochondrial membrane potential. The release of cytochrome $c$ into cytosol triggers the activation of caspase- 3 through the formation of the apoptosome [23]. Anti-cancer drug-induced caspase activation may be initiated through an extrinsic pathway or the intrinsic mitochondrial pathway [24]. Caspases play an essential role in apoptotic signaling pathways. Caspases are of two types; initiator caspases and executioner caspases. The initiator caspases (caspase-2, -8, -9, and -10) are responsible for the conversion of the inactive form of the executor caspase into the active form. Executioner caspases (caspase-3, -6, and -7) directly trigger the apoptotic process in cells [25]. For example, caspase-8 induces cell death through caspase-3 activation, an important primary caspase in the intrinsic and extrinsic pathways of apoptosis [26]. Mitochondrial damage resulting from drug-induced apoptosis is often accompanied by the release of cytosolic cytochrome $c$ from the mitochondria. X-linked inhibitor of apoptosis protein (XIAP), a member of the inhibitor of apoptosis family of proteins, is a protein that inhibits apoptotic cell death by inhibiting caspase- $3,-7$, and -9 activations [27]. Additionally, $\mathrm{Mcl}-\mathrm{1}_{(\mathrm{L})}$, a Bcl-2 family protein that inhibits apoptosis, and cellular FLICE (Fas associated with death domain [FADD]-like interleukin-1 $\beta$-converting enzyme)-inhibitory protein (c-FLIP), an antiapoptotic protein that inhibits apoptosis receptor-mediated apoptosis signaling pathway, are important miRNA targets $[28,29]$.

Many miRNAs are involved in extrinsic signaling pathways. 
miR-7 is a specific sensitizer for TRAIL-mediated apoptosis in glioblastoma multiforme and hepatocellular carcinoma cells [30]. miR-590 is regulated via the signal transducer and activator of transcription 5 (STAT5) pathway and targets Fas ligand (FasL) to promote cell survival in human acute myelogenous leukemia cells [31]. miR-21 is a direct target of FasL in pancreatic cancer, and its ectopic expression protects cancer cells from gemcitabine-mediated apoptosis [32]. miR-20a inhibits the expression of Fas in osteosarcoma cells, thereby facilitating the survival of tumor cells and enhancing their metastatic capacity [33]. Besides, miR-146a and miR-196b inhibit Fas expression, and ectopic expression of miR$196 \mathrm{~b}$ causes rapid leukemia $[34,35]$. In addition, the expression of miR-196b-5p has been shown to possess a significant negative correlation with the expression of Fas in non-small cell lung cancer (NSCLC) [35]. miR-25, which has been observed to directly target DR4 and protect against TRAIL-mediated apoptosis, is upregulated in intrahepatic bile duct cancer [36]. FADD is regulated by miR-128a or miR-155. The expression level of miR-128a is increased in acute lymphoblastic leukemia. The ectopic expression of miR-128a enhances Fas resistance in Jurkat cells by targeting FADD, but antagonizing miR-128a sensitizes Fas-mediated apoptosis [37]. Curcumin induces A549 cell apoptosis through downregulation of miRNA-186* expression that targets caspase-10, which is homologous to caspase- $8[10,38]$. c-FLIP is a major antiapoptotic protein and an important chemotherapy resistance factor [39]. miR-708 negatively regulates the expression of c-FLIP and enhances the sensitivity of renal cancer cells to various apoptotic stimuli [40]. miR-378, miR-155, and miR-let-7a can regulate cancer cell apoptosis via target caspase-3 [41]. miR-106b is associated with the recurrence of prostate cancer and directly targets caspase-7 [42]. miR-133 and miR-24a directly suppress caspase-9 to regulate apoptosis in pancreatic cancer cells [43].

In the intrinsic apoptotic pathway, apoptotic regulator genes are also regulated by many different miRNAs. miR-365 has been shown to directly target $\mathrm{Bcl}$-2-like protein 4 (Bax) and the adaptor protein Src homology 2 domain-containing 1 (SHC1) in lung adenocarcinoma and lung cancer cell lines [44]. miR-125b is significantly downregulated in gastric cancer cells. However, overexpression of miR-125b inhibits proliferation, migration, and invasion in gastric cancer cell lines (HGC-27 and MGC-803) [45]. miR-148a and miR-204 induce apoptosis by negatively targeting Bcl-2 at the posttranscriptional level $[46,47]$. In prostate cancer cell lines, ectopic expression of miR-204 has been shown to decrease cell viability and promote apoptosis through the downregulation of $\mathrm{Bcl}-2 \mathrm{ex}-$ pression [47]. miR-608 directly targets Bcl-extra large (Bcl-xL) and significantly inhibits chordoma cell proliferation by inducing apoptosis [48]. miR-23a/b and miR-27a/b can target apoptotic protease activating factor 1 (Apaf1) and thereby control the sensitivity of neurons to apoptosis [49]. The miRNAs that regulate apoptosis are summarized in Fig. 1.

\section{The role of microRNAs in endoplasmic reticulum stress pathways}

Since the ER is involved in the localization and folding of intracellularly secreted transmembrane proteins, the accumulation of unfolded proteins in cells causes cellular stress and leads to a specific ER response called the unfolded protein response (UPR) [50]. UPR is regulated by three sensor proteins; protein kinase RNAlike ER kinase (PERK), inositol-requiring enzyme 1 (IRE1), and activating transcription factor (ATF) 6 [51]. When ER stress is increased, the sensor initiates an adaptive response, including induction of ER chaperone proteins and inhibition of protein synthesis. UPR shows a cascade pattern that activates protein synthesis inhibition and induction of ER stress-related gene expression. If the adaptive response fails to alleviate ER stress and goes beyond what the cell can tolerate, both the intrinsic and extrinsic apoptotic pathways can become activated to remove the stressed cells [52]. Players involved in apoptosis include IRE1 $1 \alpha$, PERK, and ATF6 [53]. PERK hyperactivation can upregulate the CCAAT-enhancer-binding protein homologous protein ( $\mathrm{CHOP}$, also known as growth arrest and DNA damage 153) transcription factor. CHOP inhibits the expression of antiapoptotic $\mathrm{Bcl}-2$ and upregulates proapoptotic BIM and DR5 [53]. Hyperactivated IRE1 $\alpha$ activates apoptosis signal-regulating kinase 1 (ASK1) and its downstream target c-Jun NH2-terminal kinase (JNK). Phosphorylated JNK activates proapoptotic BIM and inhibits antiapoptotic Bcl-2. ATF6 likely has proapoptotic targets such as apoptotic factors caspase-12, -9 , and -3 [54].

miR-199a-5p directly binds to the binding sites of GRP78, ATF6, and IRE1 $\alpha$ [55]. PERK has been reported to be a direct target of miR-204, which increases ER stress-induced cell death in $\beta$-cells; however, ATF6 and IRE1 $\alpha$ are not affected by miR-204 [56]. Interestingly, PERK induces miR-483 expression, which is associated with the activation of ATF4, but not CHOP [57]. A recent study showed that miR-7112-3p directly targets PERK and regulates the ER stress signaling pathway (activation of the PERK/ ATF4/CHOP/caspase cascade) and apoptosis in colorectal cancer CX-1 cells [58]. Besides, colorectal cancer tissues show high expression of miR-7112-3p [58]. The enforced expression of active ATF6 decreases the expression of miR-455 [59]. miR-702 directly regulates ATF6, which inhibits apoptosis in cultured cardiomyocytes treated with isoproterenol [60]. IRE1 1 cleaves miR-17, miR-34a, miR-96, and miR-125b during ER stress to suppress the 


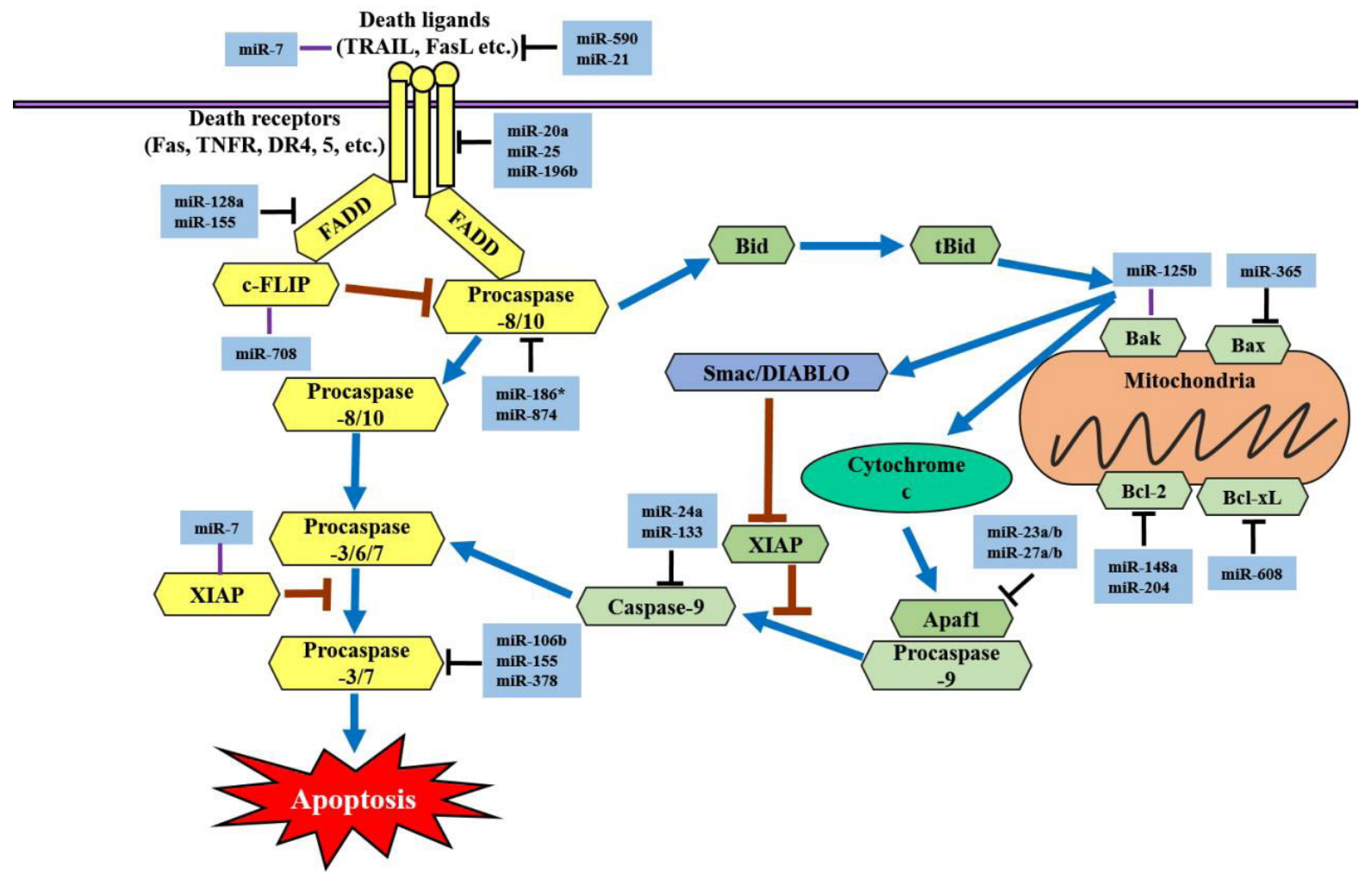

Fig. 1. MicroRNAs (miRs) that regulate the apoptotic signaling pathways. The yellow box indicates the components of the extrinsic apoptotic signaling pathway. When a death ligand binds to a death receptor (DR), an exogenous pathway is initiated. The light green box indicates the components of the intrinsic pathway. Activation of the intrinsic pathway or mitochondrial pathway releases cytochrome $\mathrm{C}$ into the cytoplasm. TRAIL, tumor necrosis factor (TNF)-related apoptosis-inducing ligand; FasL, Fas ligand; TNFR, TNF receptor 1; FADD, Fas-associated death domain protein; c-FLIP, cellular FLICE (FADD-like IL-1 $\beta$-converting enzyme)-inhibitory protein; Bid, BH3 interactingdomain death agonist; tBid, truncated Bid; Smac, second mitochondria-derived activator of caspases, also referred to as DIABLO; Bak, $\mathrm{Bcl}-2$ homologous antagonist killer; Bax, Bcl-2-like protein 4; Bcl-2, B-cell lymphoma 2; Bcl-xL, B-cell lymphoma-extra large; XIAP, X-linked inhibitor of apoptosis; Apaf1, apoptotic protease activating factor 1.

translation of proapoptotic caspase-2 [61]. ER stress-induced apoptosis is mediated via caspase-2, which is also regulated by IRE1 $\alpha$ [62]. PERK-dependent expression of miR-30c-2p inhibits $\mathrm{X}$ box-binding protein 1 (XBP1) and nuclear factor kappa-lightchain-enhancer of activated $\mathrm{B}$ cells (NF- $\mathrm{kB}$ ), and overexpression of miR-30c-2-3p exerts a proapoptotic effect by reducing the translation of XBP1 under ER stress conditions [63]. CHOP controls miR-708 transcription during prolonged ER stress [64]. miR-211 is an antiapoptotic miRNA that regulates $\mathrm{CHOP}$ expression in a PERK- and ATF4-dependent manner [65] (Fig. 2).

\section{The role of microRNAs in necroptotic cell death pathways}

Necroptosis is a programmed form of necrosis or inflammatory cell death. Even if necroptosis inhibits caspase activity, cell suicide occurs in a caspase-independent manner. When caspases are inactivated in cells, receptor-interacting protein (RIP) 1 is phosphory- lated by TNF- $\alpha$ and then interacts with RIP3 to induce necroptosis [66]. In this signaling pathway, when TNF- $\alpha$ stimulates TNFR1, TNFR1-associated DD protein (TRADD) and TNF receptor-associated factor 2 (TRAF2) send signals to receptor-interacting serine/threonine-protein kinase (RIPK) 1, finally recruiting RIPK3 to form a necrosome. The RIP1 inhibitor necrostatin-1 (nec-1) has been demonstrated to prevent the death of TNF- $\alpha$-treated FADD-deficient Jurkat cells $[67,68]$. Apoptosis-inducing factor (AIF) is transferred from the mitochondria to the cytoplasm and nucleus and induces caspase-independent chromatinolysis [69]. Necroptosis is not only associated with diseases but also with inflammatory diseases such as pancreatitis and Crohn disease [70].

Several miRNAs are known to regulate necroptosis signaling pathways. Deubiquitinase cylindromatosis (CYLD) is an important deubiquitinating enzyme involved in apoptosis or necroptosis signaling pathways. CYLD is directly targeted by miR-181b-1 and miR-19, which leads to inflammation and tumor progression [71,72]. Moreover, miR-874 and miR-512-3p have been reported 


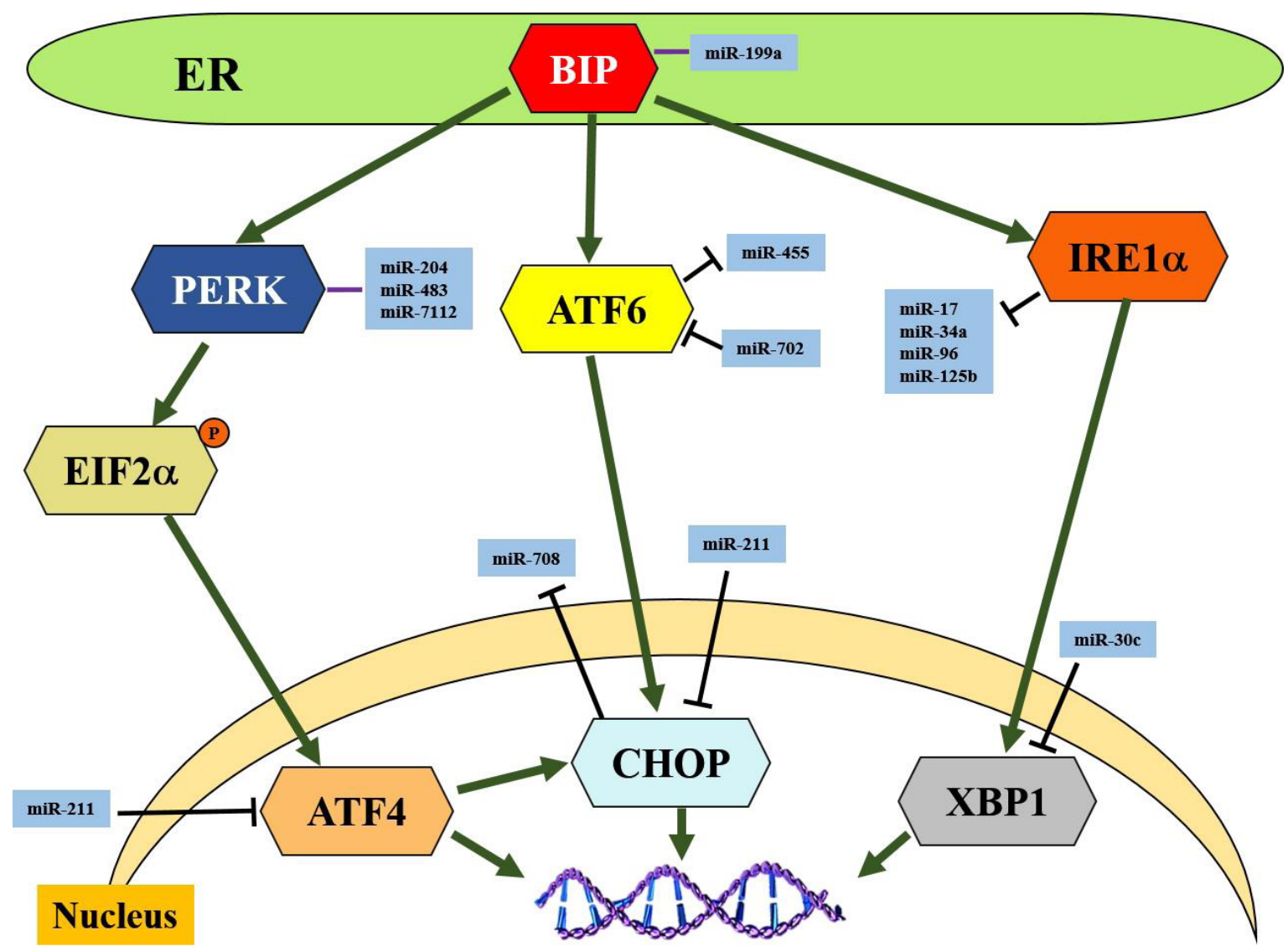

Fig. 2. MicroRNAs (miRNAs or miRs) that regulate the endoplasmic reticulum (ER) stress pathways. Various miRNAs play a role in survival or apoptosis under ER stress conditions. A critical step in unfolded protein response (UPR) signaling is the initial detection of ER stress. PERK, ATF6, and IRE 1 a possess UPR sensors that are activated by ER stress. BIP, binding immunoglobulin protein, also known as GRP78; PERK, protein kinase RNA-like ER kinase; ATF, activating transcription factor; IRE1 $\alpha$, inositol-requiring enzyme 1 alpha; ElF2 $\alpha$, eukaryotic initiation factor 2 alpha; CHOP, CCAAT-enhancer-binding protein homologous protein; XBP1, X box-binding protein 1.

to regulate necroptosis by targeting caspase- 8 , which is involved in the transition between apoptosis and necroptosis $[73,74]$. miR128 a and miR-155 regulate the activity of the adapter protein FADD $[37,75]$. miR-21 has been shown to act as a pro-necroptosis oncogene through the regulation of RIP1/3-mediated necroptosis [76]. miR-92a-3p is known to be involved in apoptosis or necrosis, which is a member of the miR-17-92 cluster. In addition, miR-92a$3 \mathrm{p}$ has been shown to significantly increase cell growth and colony formation in renal cancer cells [77] (Fig. 3).

\section{The role of microRNAs in autophagy pathways}

Autophagy is a basic catabolic process that occurs in response to starvation or other stressful conditions. Autophagy encloses dysfunctional, misfolded, or aggregated proteins and damaged cellular components (e.g., mitochondria, ER, and peroxidants) in double-membrane vesicles called autophagosomes, which eventually digest them by lysosomal enzymes and use them for cellular metabolism again [78]. Autophagy involves several key steps for the final degradation of cellular components in lysosomes; sequestration, transport to lysosomes, degradation, and utilization of degradation products. Each step is tightly regulated by evolutionarily conserved autophagy-related (ATG) genes [79].

Autophagy regulates intracellular homeostasis through the cytoplasmic turnover of proteins and organelles. In some cases, autophagy dysfunction can have various pathological consequences, including cancer, neurodegeneration, cardiovascular disorders, and microbe infection. Impaired autophagic signaling pathways are frequently observed in cancer patients. In cancer cells, autophagy has been referred to as a 'double-edged sword' because it increases apoptotic cell death [80] and maintains tumor cell survival in response to metabolic stress in vitro [81]. Since the mechanisms that regulate dual opposed survival-supporting and death-promoting roles of autophagy are still far from resolution, it seems necessary to understand the mechanisms that precisely regulate each step of 


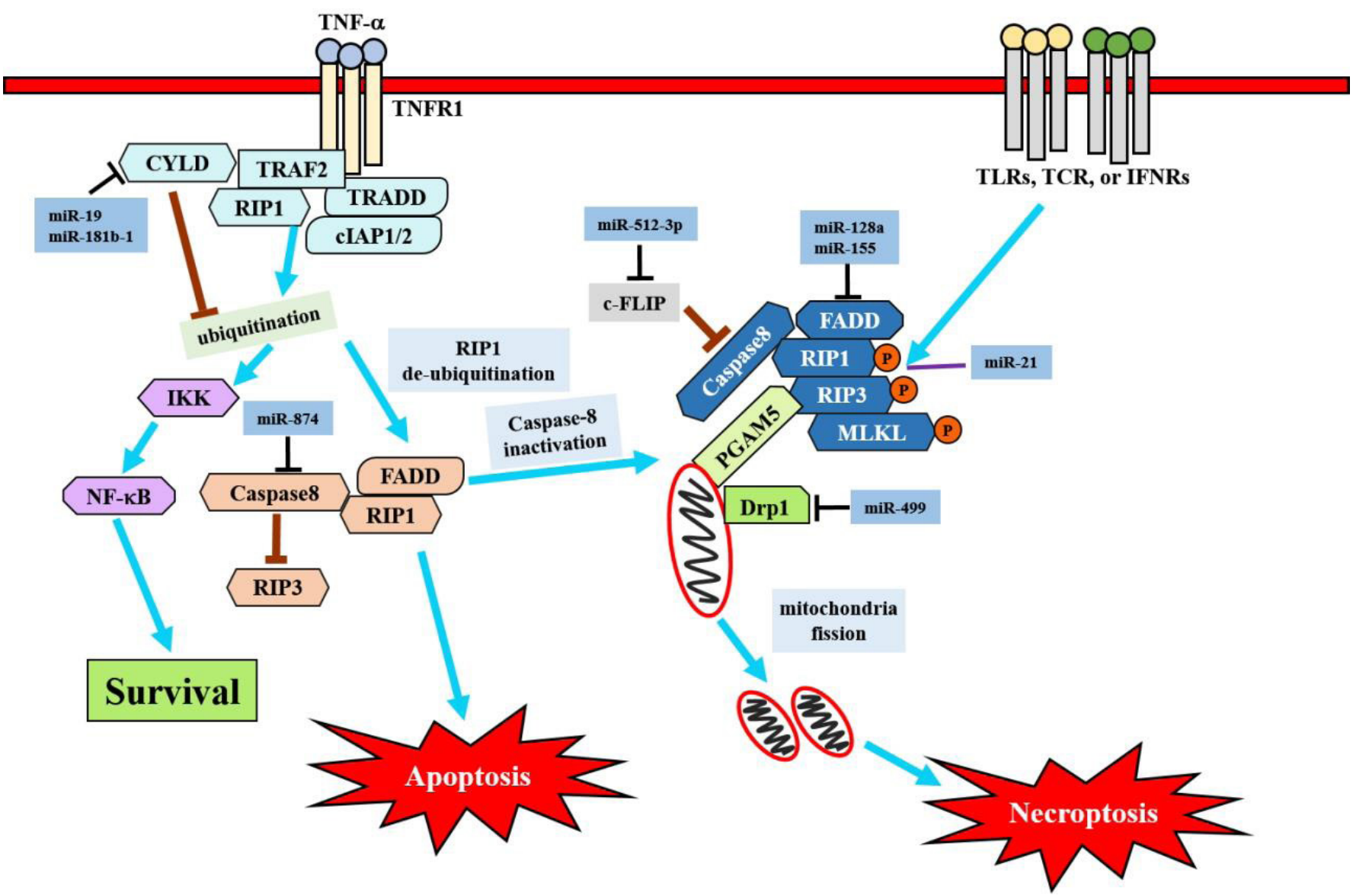

Fig. 3. MicroRNAs (miRs) that regulate necroptotic signaling pathways. Necroptosis is stimulated by TLR, IFNR, and TCR, which are members of the tumor necrosis factor (TNF) receptor superfamily. Extrisic stimili such as cellular stress, damage, and infection activated TNFR1, which can lead to cell survival, apoptosis, or necroptosis. CIAP1 and cIAP2 ubiquitinate RIP1, whereas CYLD deubiquitinates RIP1. MLKL is a critical substrate of RIP3 during the induction of necroptosis. TNFR1, TNF- $\alpha$ receptor 1 ; CYLD, cylindromatosis; TRAF2, TNF receptor-associated factor 2; RIP1, receptor-interacting protein kinase 1; TRADD, TNFR1-associated death domain protein; c-IAP1/2, cellular inhibitor of apoptosis protein 1/2; IKK, IKB kinase; NF- $\mathrm{kB}$, nuclear factor kappa-light-chain-enhancer of activated B cells; FADD, Fas-associated protein with death domain; TLRs, toll-like receptors; TCR, T-cell receptor; IFNRs, interferon receptors; c-FLIP, cellular FLICE (FADD-like interleukin-1 $\beta$-converting enzyme) inhibitory protein; MLKL, mixed lineage kinase domain-like; PGAM5, phosphoglycerate mutase 5; Drp1, dynamin-related protein 1.

autophagy to design novel intervention strategies against cancers.

One of the key downstream effectors of autophagy is the mammalian target of rapamycin (mTOR) that coordinates eukaryotic cell growth and metabolism in the presence of growth factors and abundant nutrients [82]. mTOR is well known to regulate cell growth, proliferation, metastasis, and transcription factors. It is associated with two distinct protein complexes, mTOR complex (mTORC) $1 / 2$ [83]. The three important elements of mTORC1 are mTOR, regulatory-associated protein of mTOR (Raptor), and mammalian lethal with Sec13 protein 8 (mLST8), also known as GßL. In addition, mTORC1 contains two inhibitory elements; proline-rich Akt substrate of $40 \mathrm{kDa}$ (PRAS40) and DEP domain-containing mTOR interacting protein (DEPTOR). mTORC2 contains rapamycin-insensitive companion of mTOR
(Rictor), a protein that performs similar functions in mTORC2 instead of Raptor. mTORC2 also contains DEPTOR and inhibitory elements $\mathrm{mSin} 1$ and Protor $1 / 2$. It has been found that the rapamycin complex directly inhibits mTORC1, but mTORC2 is unresponsive to rapamycin treatment $[83,84]$. Since the mTOR signaling pathway is activated in various cancers, specifically targeting the mTOR pathway via miRNA is a cancer treatment strategy. It is possible to search for various miRNAs that inhibit mTOR signaling in cancer cells, increase the effectiveness of $\mathrm{mTORC} 1 / 2$ inhibitors, and improve the effectiveness of cancer treatment.

miR-99a targets mTOR, which reduces tumorigenesis in lung cancer cells [85]. miR-193a-5p directly targets phosphoinositide-3-kinase regulatory subunit 3 (PIK3R3) and mTOR to inactivate the AKT/mTOR signaling pathway and suppress NSCLC 


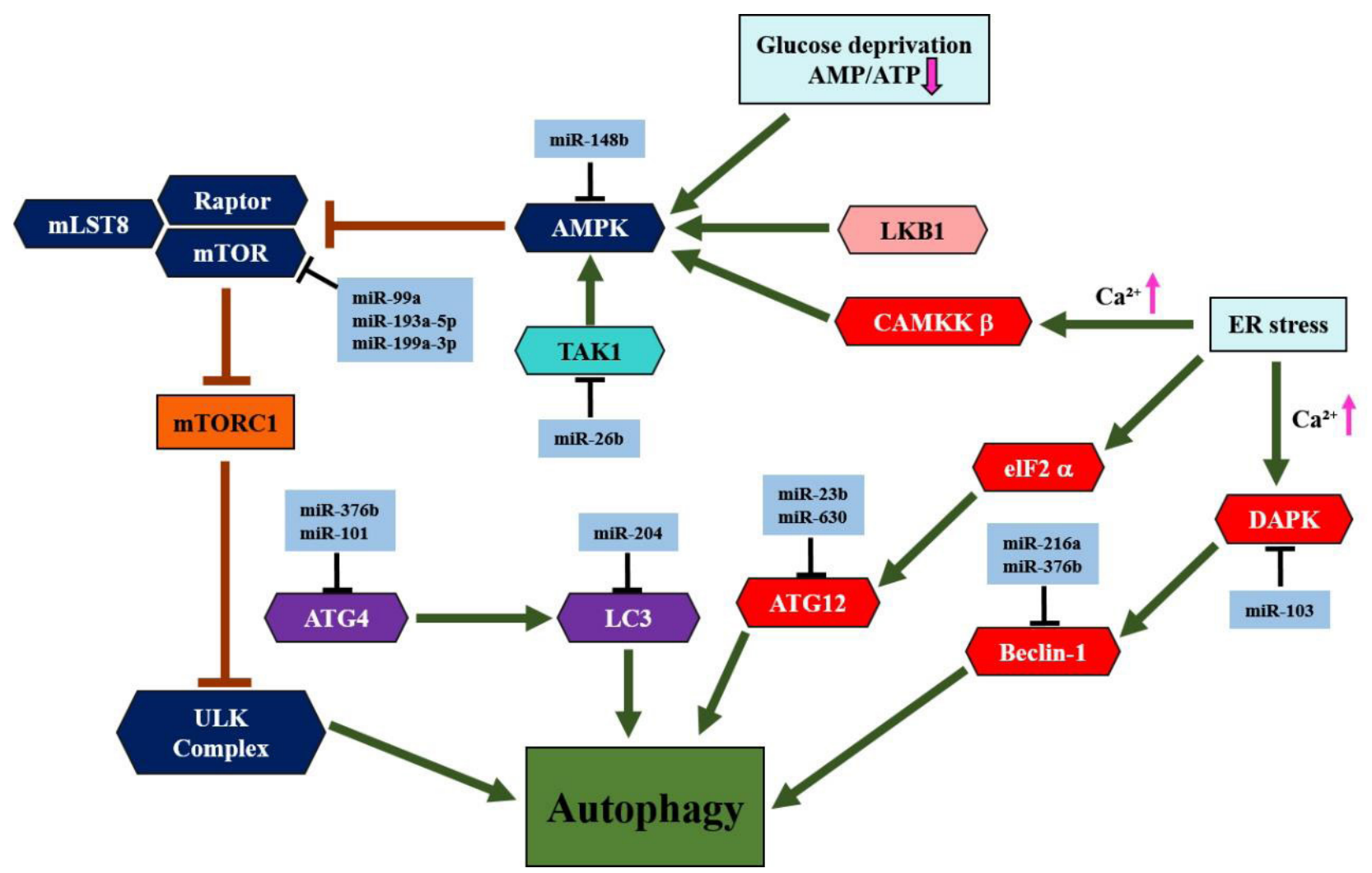

Fig. 4. miRNAs involved in the autophagy pathways. Autophagy occurs under a variety of conditions, such as cell growth signaling, glucose deficiency, hypoxia, genotoxicity, and endoplasmic reticulum (ER) stress, and activates signaling pathways that initiate or inhibit the autophagy cascade. The mTOR and AMPK proteins are important regulators of the autophagy pathway. mLST8, mammalian lethal with Sec13 protein 8, also referred to as $\mathrm{G} \beta \mathrm{L} ; \mathrm{mTOR}$, mammalian target of rapamycin; Raptor, regulatory-associated protein of mTOR; mTORC1, mTOR complex 1; ULK, Unc-51-like kinase; AMPK, AMP-activated protein kinase; TAK1, transforming growth factor $\beta$-activated kinase 1; ATG4, autophagy-related 4 cysteine peptidase; LC3, microtubule-associated proteins 1A/1B light chain 3B; AMP, adenosine monophosphate; ATP, adenosine triphosphate; LKB1, liver kinase B1; CAMKK $\beta$, calcium/calmodulin kinase kinase beta; elF2 $\alpha$, eukaryotic initiation factor 2 alpha; DAPK, death-associated protein kinase.

metastasis [86]. Moreover, miR-199a-3p regulates mTOR and c-Met to inhibit invasion and enhance doxorubicin sensitivity in hepatocarcinoma cells [87]. Downregulation of miR-148b is associated with poor survival in NSCLC [88]. In pancreatic cancer, it has been observed that miR-148b is a direct target of adenosine monophosphate-activated protein kinase (AMPK) $\alpha 1$ that inhibits cell proliferation and invasion, and enhances chemosensitivity [89]. miR-26b directly suppresses transforming growth factor $\beta$-activated kinase 1 (TAK1) and other factors to enhance chemosensitivity [90]. ATG4C, an ATG4 family member, has been observed to decrease the expression of miR-376b [91]. Moreover, miR-101 directly targets ATG4D, an ATG4 family member, which acts as a potential inhibitor of rapamycin- or etoposide-induced autophagy [92]. miR-204 interferes with autophagy and inhibits the growth of renal clear cell carcinoma [93]. In addition, miR-204 directly targets ATF2 to suppress cell proliferation and autophagy and induce apoptosis of NSCLC [94]. In pancreatic cancer cells, ATF12 expression is inhibited by miR-23b, which decreases auto- phagic activity and suppresses radio-resistance [95]. Additionally, miR-630 is considered to be a target of ATG12 [96]. Beclin-1, the main component of the class III phosphatidylinositol 3-kinase (PI3K-III) complex, is negatively regulated by miR-376b, miR30a, and miR-216a, which leads to decreased autophagic activity [91,97-99]. Death-associated protein kinase (DAPK) is a wellknown proapoptotic and suppressor of metastasis. DAPK is a direct target of miR-103 that promotes colorectal cancer metastasis [100] (Fig. 4).

\section{Conclusion}

In this review, we have summarized and discussed how miRNAs regulate apoptosis, ER stress, necroptosis, and autophagy by targeting a wide range of components of these pathways and documented the aberrant expression of miRNAs in cancer and the oncogenic or tumor-suppressor roles of miRNAs. miRNAs discussed in this review could be used as promising diagnostic and prognostic 
biomarkers as well as effective therapeutic targets for various cancers. However, further studies are needed to improve knowledge about the mechanisms and functions of miRNAs in various programmed cell death pathways in cancer cells. In addition, it is necessary to determine the best formulation and precise drug delivery system to target cancerous tissues while avoiding unwanted miRNA effects that can result from targeting important genes in other healthy tissues. Finally, the selected miRNAs should have the potential to intervene and modify cell physiology and behavior in many human pathologies, including the deregulation of the cell death machinery.

\section{Acknowledgments}

\section{Conflicts of interest}

No potential conflict of interest relevant to this article was reported.

\section{Funding}

This study was supported by the Basic Science Research Program through the National Research Foundation of Korea (NRF) funded by the Ministry of Education (2020R1F1A1048259).

\section{Author contributions}

Conceptualization: all authors; Funding acquisition, Project administration, Validation: TJL; Investigation: JHJ; Writing-original draft: JHJ; Writing-review \& editing: TJL.

\section{ORCID}

Ji Hoon Jang, https://orcid.org/0000-0001-5272-2441

Tae-Jin Lee, https://orcid.org/0000-0003-3934-2035

\section{References}

1. Mohr AM, Mott JL. Overview of microRNA biology. Semin Liver Dis 2015;35:3-11.

2. Liu B, Li J, Cairns MJ. Identifying miRNAs, targets and functions. Brief Bioinform 2014;15:1-19.

3. Acunzo M, Romano G, Wernicke D, Croce CM. MicroRNA and cancer: a brief overview. Adv Biol Regul 2015;57:1-9.

4. van den Berg A, Mols J, Han J. RISC-target interaction: cleavage and translational suppression. Biochim Biophys Acta 2008;1779:668-77.

5. Rajewsky N. MicroRNA target predictions in animals. Nat Genet 2006;38(Suppl):S8-13.

6. Mazière P, Enright AJ. Prediction of microRNA targets. Drug Discov Today 2007;12:452-8.
7. Huntzinger E, Izaurralde E. Gene silencing by microRNAs: contributions of translational repression and mRNA decay. Nat Rev Genet 2011;12:99-110.

8. Jo MH, Shin S, Jung SR, Kim E, Song JJ, Hohng S. Human Argonaute 2 has diverse reaction pathways on target RNAs. Mol Cell 2015;59:117-24.

9. Grammatikakis I, Gorospe M, Abdelmohsen K. Modulation of cancer traits by tumor suppressor microRNAs. Int J Mol Sci 2013;14:1822-42.

10. Su Z, Yang Z, Xu Y, Chen Y, Yu Q. MicroRNAs in apoptosis, autophagy and necroptosis. Oncotarget 2015;6:8474-90.

11. Di Leva G, Garofalo M, Croce CM. MicroRNAs in cancer. Annu Rev Pathol 2014;9:287-314.

12. Ali Syeda Z, Langden SS, Munkhzul C, Lee M, Song SJ. Regulatory mechanism of microRNA expression in cancer. Int J Mol Sci 2020;21:1723.

13. Lu J, Getz G, Miska EA, Alvarez-Saavedra E, Lamb J, Peck D, et al. MicroRNA expression profiles classify human cancers. Nature 2005;435:834-8.

14. Lambertz I, Nittner D, Mestdagh P, Denecker G, Vandesompele J, Dyer MA, et al. Monoallelic but not biallelic loss of Dicer 1 promotes tumorigenesis in vivo. Cell Death Differ 2010;17: $633-41$.

15. Kumar MS, Pester RE, Chen CY, Lane K, Chin C, Lu J, et al. Dicer1 functions as a haploinsufficient tumor suppressor. Genes Dev 2009;23:2700-4.

16. Elmore S. Apoptosis: a review of programmed cell death. Toxicol Pathol 2007;35:495-516.

17. Melet A, Song K, Bucur O, Jagani Z, Grassian AR, Khosravi-Far R. Apoptotic pathways in tumor progression and therapy. Adv Exp Med Biol 2008;615:47-79.

18. Fulda S, Debatin KM. Targeting inhibitor of apoptosis proteins (LAPs) for diagnosis and treatment of human diseases. Recent Pat Anticancer Drug Discov 2006;1:81-9.

19. Green DR, Llambi F. Cell death signaling. Cold Spring Harb Perspect Biol 2015;7:a006080.

20. Lavrik I, Golks A, Krammer PH. Death receptor signaling. J Cell Sci 2005;118(Pt2):265-7.

21. Debatin KM, Krammer PH. Death receptors in chemotherapy and cancer. Oncogene 2004;23:2950-66.

22. Gerspach J, Pfizenmaier K, Wajant H. Therapeutic targeting of CD95 and the TRAIL death receptors. Recent Pat Anticancer Drug Discov 2011;6:294-310.

23. Garrido C, Galluzzi L, Brunet M, Puig PE, Didelot C, Kroemer G. Mechanisms of cytochrome c release from mitochondria. Cell Death Differ 2006;13:1423-33.

24. Fulda S, Debatin KM. Targeting apoptosis pathways in cancer 
therapy. Curr Cancer Drug Targets 2004;4:569-76.

25. Lamkanfi M, Declercq W, Kalai M, Saelens X, Vandenabeele P. Alice in caspase land. A phylogenetic analysis of caspases from worm to man. Cell Death Differ 2002;9:358-61.

26. Walters J, Pop C, Scott FL, Drag M, Swartz P, Mattos C, et al. A constitutively active and uninhibitable caspase-3 zymogen efficiently induces apoptosis. Biochem J 2009;424:335-45.

27. Deveraux QL, Takahashi R, Salvesen GS, Reed JC. X-linked IAP is a direct inhibitor of cell-death proteases. Nature 1997; 388:300-4.

28. Bae J, Donigian JR, Hsueh AJ. Tankyrase 1 interacts with Mcl-1 proteins and inhibits their regulation of apoptosis. J Biol Chem 2003;278:5195-204.

29. Natoni F, Diolordi L, Santoni C, Gilardini Montani MS. Sodium butyrate sensitises human pancreatic cancer cells to both the intrinsic and the extrinsic apoptotic pathways. Biochim Biophys Acta 2005; 1745:318-29.

30. Zhang X, Zhang X, Hu S, Zheng M, Zhang J, Zhao J, et al. Identification of miRNA-7 by genome-wide analysis as a critical sensitizer for TRAIL-induced apoptosis in glioblastoma cells. Nucleic Acids Res 2017;45:5930-44.

31. Favreau AJ, Shaffiey F, Cross E, Sathyanarayana P. Mir-590 is a novel STAT5 regulated oncogenic miRNA and targets FasL in acute myeloid leukemia. Blood 2013;122:3811.

32. Wang P, Zhuang L, Zhang J, Fan J, Luo J, Chen H, et al. The serum miR-21 level serves as a predictor for the chemosensitivity of advanced pancreatic cancer, and miR-21 expression confers chemoresistance by targeting FasL. Mol Oncol 2013;7:334-45.

33. Huang G, Nishimoto K, Zhou Z, Hughes D, Kleinerman ES. miR-20a encoded by the miR-17-92 cluster increases the metastatic potential of osteosarcoma cells by regulating Fas expression. Cancer Res 2012;72:908-16.

34. Curtale G, Citarella F, Carissimi C, Goldoni M, Carucci N, Fulci $\mathrm{V}$, et al. An emerging player in the adaptive immune response: microRNA-146a is a modulator of IL-2 expression and activation-induced cell death in T lymphocytes. Blood 2010;115: 265-73.

35. Huang X, Xiao S, Zhu X, Yu Y, Cao M, Zhang X, et al. miR196b-5p-mediated downregulation of FAS promotes NSCLC progression by activating IL6-STAT3 signaling. Cell Death Dis 2020;11:785.

36. Razumilava N, Bronk SF, Smoot RL, Fingas CD, Werneburg NW, Roberts LR, et al. miR-25 targets TNF-related apoptosis inducing ligand (TRAIL) death receptor-4 and promotes apoptosis resistance in cholangiocarcinoma. Hepatology 2012;55: 465-75.

37. Yamada N, Noguchi S, Kumazaki M, Shinohara H, Miki K,
Naoe T, et al. Epigenetic regulation of microRNA-128a expression contributes to the apoptosis-resistance of human T-cell leukaemia jurkat cells by modulating expression of fas-associated protein with death domain (FADD). Biochim Biophys Acta 2014;1843:590-602.

38. Zhang J, Du Y, Wu C, Ren X, Ti X, Shi J, et al. Curcumin promotes apoptosis in human lung adenocarcinoma cells through miR-186* signaling pathway. Oncol Rep 2010;24:1217-23.

39. Fulda S. Targeting c-FLICE-like inhibitory protein (CFLAR) in cancer. Expert Opin Ther Targets 2013;17:195-201.

40. Kim EA, Kim SW, Nam J, Sung EG, Song IH, Kim JY, et al. Inhibition of c-FLIPL expression by miRNA-708 increases the sensitivity of renal cancer cells to anti-cancer drugs. Oncotarget 2016;7:31832-46.

41. Czochor JR, Glazer PM. MicroRNAs in cancer cell response to ionizing radiation. Antioxid Redox Signal 2014;21:293-312.

42. Yin W, Chen J, Wang G, Zhang D. MicroRNA-106b functions as an oncogene and regulates tumor viability and metastasis by targeting LARP4B in prostate cancer. Mol Med Rep 2019;20: 951-8.

43. Park JK, Doseff AI, Schmittgen TD. MicroRNAs targeting caspase-3 and -7 in PANC-1 cells. Int J Mol Sci 2018;19:1206.

44. Wang Y, Zhang S, Bao H, Mu S, Zhang B, Ma H, et al. MicroRNA-365 promotes lung carcinogenesis by downregulating the USP33/SLIT2/ROBO1 signalling pathway. Cancer Cell Int 2018;18:64.

45. Zhang X, Yao J, Guo K, Huang H, Huai S, Ye R, et al. The functional mechanism of miR-125b in gastric cancer and its effect on the chemosensitivity of cisplatin. Oncotarget 2017;9:210519.

46. Li Q, Ren P, Shi P, Chen Y, Xiang F, Zhang L, et al. MicroRNA-148a promotes apoptosis and suppresses growth of breast cancer cells by targeting B-cell lymphoma 2. Anticancer Drugs 2017;28:588-95.

47. Lin YC, Lin JF, Tsai TF, Chou KY, Chen HE, Hwang TI. Tumor suppressor miRNA-204-5p promotes apoptosis by targeting BCL2 in prostate cancer cells. Asian J Surg 2017;40:396-406.

48. Zhang Y, Schiff D, Park D, Abounader R. MicroRNA-608 and microRNA-34a regulate chordoma malignancy by targeting EGFR, Bcl-xL and MET. PLoS One 2014;9:e91546.

49. Chen Q, Xu J, Li L, Li H, Mao S, Zhang F, et al. MicroRNA-23a/b and microRNA-27a/b suppress Apaf-1 protein and alleviate hypoxia-induced neuronal apoptosis. Cell Death Dis 2014;5:e1132.

50. English AR, Voeltz GK. Endoplasmic reticulum structure and interconnections with other organelles. Cold Spring Harb Perspect Biol 2013;5:a013227. 
51. Corazzari M, Gagliardi M, Fimia GM, Piacentini M. Endoplasmic reticulum stress, unfolded protein response, and cancer cell fate. Front Oncol 2017;7:78.

52. Szegezdi E, Logue SE, Gorman AM, Samali A. Mediators of endoplasmic reticulum stress-induced apoptosis. EMBO Rep 2006;7:880-5.

53. Oakes SA, Papa FR. The role of endoplasmic reticulum stress in human pathology. Annu Rev Pathol 2015;10:173-94.

54. Yu B, Wen L, Xiao B, Han F, Shi Y. Single prolonged stress induces ATF6 alpha-dependent endoplasmic reticulum stress and the apoptotic process in medial frontal cortex neurons. BMC Neurosci 2014;15:115.

55. Zhou Y, Jia WK, Jian Z, Zhao L, Liu CC, Wang Y, et al. Downregulation of microRNA-199a-5p protects cardiomyocytes in cyanotic congenital heart disease by attenuating endoplasmic reticulum stress. Mol Med Rep 2017;16:2992-3000.

56. Xu G, Chen J, Jing G, Grayson TB, Shalev A. miR-204 targets PERK and regulates UPR signaling and $\beta$-cell apoptosis. Mol Endocrinol 2016;30:917-24.

57. Hiramatsu N, Chiang K, Aivati C, Rodvold JJ, Lee JM, Han J, et al. PERK-mediated induction of microRNA-483 disrupts cellular ATP homeostasis during the unfolded protein response. J Biol Chem 2020;295:237-49.

58. Kong F, Zou H, Liu X, He J, Zheng Y, Xiong L, et al. miR-7112$3 p$ targets PERK to regulate the endoplasmic reticulum stress pathway and apoptosis induced by photodynamic therapy in colorectal cancer CX-1 cells. Photodiagnosis Photodyn Ther 2020;29:101663.

59. Byrd AE, Brewer JW. Micro(RNA)managing endoplasmic reticulum stress. IUBMB Life 2013;65:373-81.

60. Zhang WG, Chen L, Dong Q, He J, Zhao HD, Li FL, et al. Mmu-miR-702 functions as an anti-apoptotic mirtron by mediating ATF6 inhibition in mice. Gene 2013;531:235-42.

61. Upton JP, Wang L, Han D, Wang ES, Huskey NE, Lim L, et al. IRE1 $a$ cleaves select microRNAs during ER stress to derepress translation of proapoptotic caspase-2. Science 2012;338:81822.

62. Upton JP, Austgen K, Nishino M, Coakley KM, Hagen A, Han $\mathrm{D}$, et al. Caspase- 2 cleavage of BID is a critical apoptotic signal downstream of endoplasmic reticulum stress. Mol Cell Biol 2008;28:3943-51.

63. Byrd AE, Aragon IV, Brewer JW. MicroRNA-30c-2* limits expression of proadaptive factor XBP1 in the unfolded protein response. J Cell Biol 2012;196:689-98.

64. Behrman S, Acosta-Alvear D, Walter P. A CHOP-regulated microRNA controls rhodopsin expression. J Cell Biol 2011;192: 919-27.
65. Chitnis NS, Pytel D, Bobrovnikova-Marjon E, Pant D, Zheng $\mathrm{H}$, Maas NL, et al. miR-211 is a prosurvival microRNA that regulates chop expression in a PERK-dependent manner. Mol Cell 2012;48:353-64.

66. Grootjans S, Vanden Berghe T, Vandenabeele P. Initiation and execution mechanisms of necroptosis: an overview. Cell Death Differ 2017;24:1184-95.

67. Liu Y, Liu T, Lei T, Zhang D, Du S, Girani L, et al. RIP1/RIP3regulated necroptosis as a target for multifaceted disease therapy (Review). Int J Mol Med 2019;44:771-86.

68. MacEwan DJ. TNF ligands and receptors: a matter of life and death. Br J Pharmacol 2002;135:855-75.

69. Baritaud M, Cabon L, Delavallée L, Galán-Malo P, Gilles ME, Brunelle-Navas MN, et al. AIF-mediated caspase-independent necroptosis requires ATM and DNA- PK-induced histone H2AX Ser139 phosphorylation. Cell Death Dis 2012;3:e390.

70. Dhuriya YK, Sharma D. Necroptosis: a regulated inflammatory mode of cell death. J Neuroinflammation 2018;15:199.

71. Andalib A, Rashed S, Dehbashi M, Hajati J, Noorbakhsh F, Ganjalikhani-Hakemi M. The upregulation of hsa-mir-181b-1 and downregulation of its target CYLD in the late-stage of tumor progression of breast cancer. Indian J Clin Biochem 2020; $35: 312-21$.

72. Ye H, Liu X, Lv M, Wu Y, Kuang S, Gong J, et al. MicroRNA and transcription factor co-regulatory network analysis reveals miR19 inhibits CYLD in T-cell acute lymphoblastic leukemia. Nucleic Acids Res 2012;40:5201-14.

73. Wang K, Liu F, Zhou LY, Ding SL, Long B, Liu CY, et al. miR874 regulates myocardial necrosis by targeting caspase-8. Cell Death Dis 2013;4:e709.

74. Chen F, Zhu HH, Zhou LF, Wu SS, Wang J, Chen Z. Inhibition of c-FLIP expression by miR-512-3p contributes to taxol-induced apoptosis in hepatocellular carcinoma cells. Oncol Rep 2010;23:1457-62.

75. Wang HQ, Yu XD, Liu ZH, Cheng X, Samartzis D, Jia LT, et al. Deregulated miR-155 promotes Fas-mediated apoptosis in human intervertebral disc degeneration by targeting FADD and caspase-3. J Pathol 2011;225:232-42.

76. Ma X, Conklin DJ, Li F, Dai Z, Hua X, Li Y, et al. The oncogenic microRNA miR-21 promotes regulated necrosis in mice. Nat Commun 2015;6:7151.

77. Zeng R, Huang J, Sun Y, Luo J. Cell proliferation is induced in renal cell carcinoma through miR-92a-3p upregulation by targeting FBXW7. Oncol Lett 2020;19:3258-68.

78. Saha S, Panigrahi DP, Patil S, Bhutia SK. Autophagy in health and disease: a comprehensive review. Biomed Pharmacother 2018;104:485-95. 
79. Yang Z, Klionsky DJ. An overview of the molecular mechanism of autophagy. Curr Top Microbiol Immunol 2009;335: $1-32$.

80. Colell A, Ricci JE, Tait S, Milasta S, Maurer U, Bouchier-Hayes $\mathrm{L}$, et al. GAPDH and autophagy preserve survival after apoptotic cytochrome $\mathrm{c}$ release in the absence of caspase activation. Cell 2007;129:983-97.

81. Degenhardt K, Mathew R, Beaudoin B, Bray K, Anderson D, Chen $\mathrm{G}$, et al. Autophagy promotes tumor cell survival and restricts necrosis, inflammation, and tumorigenesis. Cancer Cell 2006; 10:51-64.

82. Iwamaru A, Kondo Y, Iwado E, Aoki H, Fujiwara K, Yokoyama $\mathrm{T}$, et al. Silencing mammalian target of rapamycin signaling by small interfering RNA enhances rapamycin-induced autophagy in malignant glioma cells. Oncogene 2007;26:1840-51.

83. Saxton RA, Sabatini DM. mTOR signaling in growth, metabolism, and disease. Cell 2017;168:960-76.

84. Rabanal-Ruiz Y, Otten EG, Korolchuk VI. mTORC1 as the main gateway to autophagy. Essays Biochem 2017;61:565-84.

85. Yin H, Ma J, Chen L, Piao S, Zhang Y, Zhang S, et al. miR-99a enhances the radiation sensitivity of non-small cell lung cancer by targeting mTOR. Cell Physiol Biochem 2018;46:471-81.

86. Yu T, Li J, Yan M, Liu L, Lin H, Zhao F, et al. MicroRNA-193a$3 p$ and $-5 p$ suppress the metastasis of human non-small-cell lung cancer by downregulating the ERBB4/PIK3R3/mTOR/ S6K2 signaling pathway. Oncogene 2015;34:413-23.

87. Callegari E, D'Abundo L, Guerriero P, Simioni C, Elamin BK, Russo M, et al. miR-199a-3p modulates MTOR and PAK4 pathways and inhibits tumor growth in a hepatocellular carcinoma transgenic mouse model. Mol Ther Nucleic Acids 2018; 11:485-93.

88. Ge H, Li B, Hu WX, Li RJ, Jin H, Gao MM, et al. MicroRNA-148b is down-regulated in non-small cell lung cancer and associated with poor survival. Int J Clin Exp Pathol 2015;8: $800-5$.

89. Zhao G, Zhang JG, Liu Y, Qin Q, Wang B, Tian K, et al. miR$148 \mathrm{~b}$ functions as a tumor suppressor in pancreatic cancer by targeting AMPKa1. Mol Cancer Ther 2013;12:83-93.
90. Li H, Wang Y, Song Y. MicroRNA-26b inhibits the immune response to Mycobacterium tuberculosis (M.tb) infection in THP-1 cells via targeting TGF $\beta$-activated kinase- 1 (TAK1), a promoter of the NF- $\kappa$ B pathway. Int J Clin Exp Pathol 2018; 11:1218-27.

91. Korkmaz G, le Sage C, Tekirdag KA, Agami R, Gozuacik D. miR-376b controls starvation and mTOR inhibition-related autophagy by targeting ATG4C and BECN1. Autophagy 2012;8:165-76.

92. Fu Z, Luo W, Wang J, Peng T, Sun G, Shi J, et al. Malat1 activates autophagy and promotes cell proliferation by sponging miR-101 and upregulating STMN1, RAB5A and ATG4D expression in glioma. Biochem Biophys Res Commun 2017;492: $480-6$.

93. Mikhaylova O, Stratton Y, Hall D, Kellner E, Ehmer B, Drew AF, et al. VHL-regulated MiR-204 suppresses tumor growth through inhibition of LC3B-mediated autophagy in renal clear cell carcinoma. Cancer Cell 2012;21:532-46.

94. Zhang S, Gao L, Thakur A, Shi P, Liu F, Feng J, et al. miRNA-204 suppresses human non-small cell lung cancer by targeting ATF2. Tumour Biol 2016;37:11177-86.

95. An Y, Zhang Z, Shang Y, Jiang X, Dong J, Yu P, et al. miR-23b$3 p$ regulates the chemoresistance of gastric cancer cells by targeting ATG12 and HMGB2. Cell Death Dis 2015;6:e1766.

96. Gozuacik D, Akkoc Y, Ozturk DG, Kocak M. Autophagy-regulating microRNAs and cancer. Front Oncol 2017;7:65.

97. Sun T, Li MY, Li PF, Cao JM. MicroRNAs in cardiac autophagy: small molecules and big role. Cells 2018;7:104.

98. Zhao Y, Wang Z, Zhang W, Zhang L. MicroRNAs play an essential role in autophagy regulation in various disease phenotypes. Biofactors 2019;45:844-56.

99. Menghini R, Casagrande V, Marino A, Marchetti V, Cardellini M, Stoehr R, et al. miR-216a: a link between endothelial dysfunction and autophagy. Cell Death Dis 2014;5:e1029.

100. Chen HY, Lin YM, Chung HC, Lang YD, Lin CJ, HuangJ, et al. miR-103/107 promote metastasis of colorectal cancer by targeting the metastasis suppressors DAPK and KLF4. Cancer Res 2012;72:3631-41. 